\title{
Handling of uncertainty in life cycle inventory by correlated multivariate lognormal distributions: application to the design of supply chain networks
}

\author{
Juan A. Reyes-Labarta ${ }^{a}$, Raquel Salcedo-Díaz ${ }^{\mathrm{a}}$, Rubén Ruiz-Femeniaa ${ }^{\mathrm{a}}$, Gonzalo \\ Guillén-Gosálbez $^{\mathrm{b}^{*}}$, Jose A. Caballero ${ }^{\mathrm{a}}$ \\ ${ }^{a}$ Department of Chemical Engineering, University of Alicante. Ap. Correos 99, E- \\ 03080, Alicante. Spain. \\ ${ }^{b}$ Departament d'Enginyeria Química, Universitat Rovira i Virgili, Tarragona E-43007, \\ Spain. \\ gonzalo.guillen@urv.cat
}

\begin{abstract}
In this work, we analyze the effect of incorporating life cycle inventory (LCI) uncertainty on the multi-objective optimization of chemical supply chains (SC) considering simultaneously their economic and environmental performance. To this end, we present a stochastic multi-scenario mixed-integer linear programming (MILP) coupled with a two-step transformation scenario generation algorithm with the unique feature of providing scenarios where the LCI random variables are correlated and each one of them has the desired lognormal marginal distribution. The environmental performance is quantified following life cycle assessment (LCA) principles, which are represented in the model formulation through standard algebraic equations. The capabilities of our approach are illustrated through a case study of a petrochemical supply chain. We show that the stochastic solution improves the economic performance of the SC in comparison with the deterministic one at any level of the environmental impact, and moreover the correlation among environmental burdens provides more realistic scenarios for the decision making process.
\end{abstract}

Keywords: lognormal distribution, life cycle assessment, multi-objective optimization, sustainable supply chain, risk management.

\section{Introduction}

Environmental databases coupled with mathematical programming techniques have enhanced tremendously the quantitative assessment of the environmental performance of process system engineering designs. The databases offer detailed information with a huge list of environmental burdens for each source of environmental impact in the system (e.g., utilities, raw materials or transport). Nevertheless, the values listed in the environmental database should be used with caution because their value cannot be considered as perfectly known. As the environmental assessment relies on these values, incorporating uncertainty through stochastic optimization guarantees a better performance of the system in comparison with the behavior exhibited for the same system but designed with deterministic optimization, which assumes nominal values for the uncertain parameters thus neglecting their variability (Grossmann and GuillénGosálbez, 2010). A case where is specially important the incorporation of uncertainty, is the design of a supply chain. Ruiz-Femenia et al. (2013), studied the effect of the demand uncertainty in the optimal design of a petrochemical supply chain considering 
simultaneously their economic and environmental performance. There are also studies incorporating uncertainty in the price of the $\mathrm{CO}_{2}$ emissions rights in the design of a supply chain, located in a region where carbon trading is promoted by the government (Ruiz-Femenia et al., 2012).

To handle uncertainty, a Monte Carlo simulation can be performed. One of the decisions for a Monte Carlo simulation is the choice of probability distributions for the random variables. Selecting a distribution for each individual variable is often straightforward, but deciding what dependencies should exist among the variables is not obvious. However, there is a scarcity of information on which to base the dependence among environmental burdens. Therefore, it is useful to investigate with different correlating combinations among the environmental burdens emissions, gathered in the life cycle inventory (LCI), in order to determine how the model faces uncertainty, which is described with a set of realistic scenarios from a correlated distribution.

In this work, we explore the effect of the uncertainty in the life cycle inventory (LCI) for the optimal design of a supply chain (SC) considering simultaneously its economic and environmental performance, which is quantified following life cycle assessment (LCA) principles that consider the greenhouse gas (GHG) emissions released in all the echelons of the SC.

\section{Methodology}

We model the uncertainty in the LCI by a set of scenarios, generated by Monte Carlo sampling. Using the lognormal distribution, which is the default distribution for Ecoinvent database (Goedkoop et al., 1998), we implement a two-step transformation algorithm to generate the scenarios from a correlated multivariate random distribution function with the desired lognormal marginal distribution for each environmental burden in the LCI. Given are the mean expected value, the geometric standard deviation of each burden and the correlation matrix (whose entries range from -1 to 1 , and 0 means no correlation). The geometric standard deviation is calculated from the uncertainty factors in conjunction with the pedigree matrix (Frischknecht et al., 2005). With these three inputs we compute the covariance matrix, whose diagonal elements contain the variances for each variable, while the off-diagonal elements contain the covariance among variables. Then, we use the probability density function of a multivariate normal distribution to generate a certain number of scenarios. Thus, a statistical dependence among each pair of variables is created, following each variable a normal marginal distribution (Figure 1a). Then we apply the 2 step algorithm:

Step 1: Apply a transformation separately to each random variable (i.e., environmental burden) using the normal cumulative distribution function with the corresponding expected mean and standard deviation. This transformation results in a correlated distribution with uniform marginal distribution on the interval $(0,1)$ (Figure $1 b)$.

Step 2: Transform each random variable (i.e., each burden) applying the inverse cumulative distribution function of a lognormal distribution (which is the desired marginal distribution). This transformation creates correlated random variables whose marginal distributions are exactly the lognormal distribution (Figure 1c). 


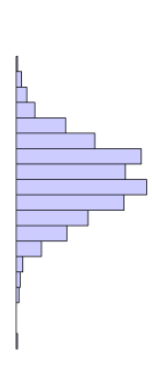

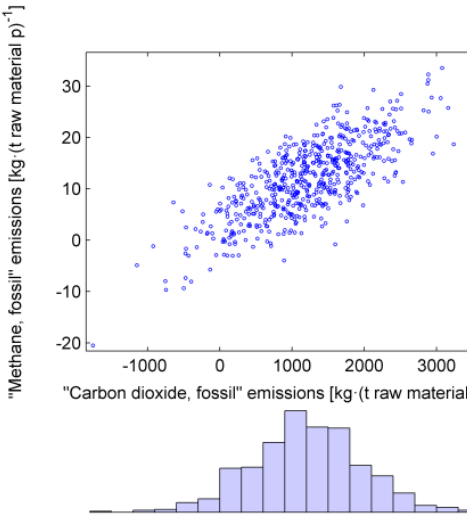

a)

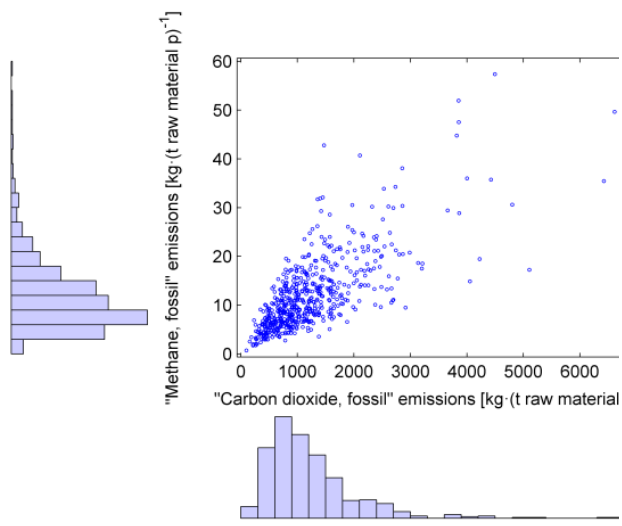

c)

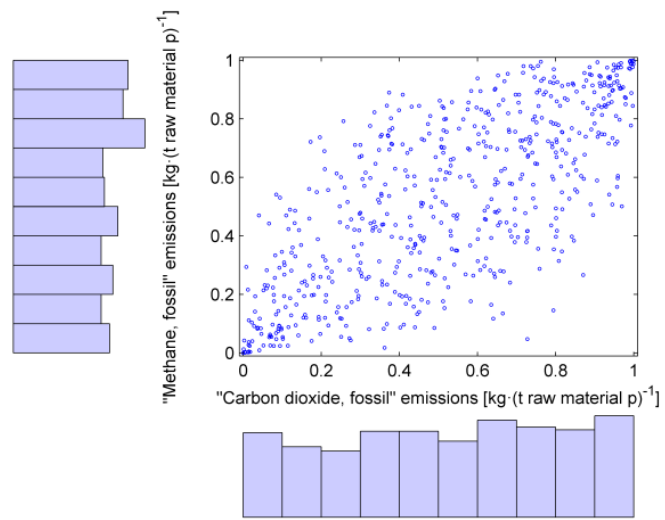

b)

Figure 1. Correlated bivariate distributions with marginal: a) normal; b) uniform; c) lognormal distributions for two environmental burdens in the life cycle inventory, carbon dioxide and methane, for one $\mathrm{kg}$ of the raw material propylene.

\section{Mathematical model}

The MILP formulation is based on that introduced by Guillén-Gosálbez and Grossmann (2009) for the case of petrochemical SC . It includes binary variables to represent the occurrence of the capacity expansion of manufacturing technology $i$ at plant $j$ in time period $t\left(X_{i j t}^{P L}\right)$, the capacity expansion of warehouse $k$ in time period $t\left(X_{k t}^{W H}\right)$, and the establishment of transportation links between plant $j$ and warehouse $k$ in time period $t\left(Y_{j k t}^{P L}\right)$, whereas continuous ones denote the transportation flows, capacity expansions, storage inventories and production rates.

The model includes three main blocks of equations: mass balances, capacity constraints and objective functions. A brief outline of each of these sets of equations is next given.

3.1. Mass balance equations

The mass balance must be satisfied for each node embedded in the network. Thus, for each plant $j$ and chemical $p$, the purchases in period $t\left(P U_{j p t}\right)$ plus the amount 
produced must equal the amount transported from the plant to the warehouses $\left(Q_{j k p t}^{P L}\right)$ plus the amount consumed:

$$
P U_{j p t}+\sum_{i \in \mathrm{OUT}(p)} W_{i j p t}=\sum_{k} Q_{j k p t}^{P L}+\sum_{i \in I N(p)} W_{i j p t} \quad \forall j, p, t
$$

\subsection{Capacity constraints}

Plant and warehouses capacity expansions are bounded between upper and lower limits, using the binary variables $\left(X_{i j t}^{P L}\right)$ and $\left(X_{k t}^{W H}\right)$ to allow or prevent the expansion.

Analogously, regarding the transportation flows, a zero value of the binary variable $\left(Y_{j k t}^{P L}\right)$ prevents the flow of materials between the corresponding nodes, whereas a value of one allows the materials flow within some lower and upper bounds.

\subsection{Objective functions}

\subsubsection{Economic performance}

The economic objective is represented by the net present value (NPV), which is calculated as the summation of the discounted cash flows (CFt) generated in each of the time periods $t$ in which the time horizon is divided.

\subsubsection{Environmental performance}

The environmental performance of the SC is assessed according to the principles of Life Cycle Assessment (LCA) using the global warming potential (GWP) indicator as described by the IPCC 2007 (Intergovernmental Panel on Climate Change) (Hischier R., 2010). The probability of meeting unfavorable scenarios is controlled by adding the worst case (WC) environmental impact as an additional objective to be minimized. The worst case is determined from the maximum GWP attained over all the scenarios as follows:

$$
\mathrm{GWP}_{s} \leq W C \quad \forall s
$$

\subsection{Solution method}

The solution to this problem is given by a set of Pareto alternatives representing the optimal trade-off between the two objectives. In this work, these Pareto solutions are determined via the $\varepsilon$-constraint method (Ehrgott, 2005), which entails solving a set of instances of the single-objective NPV for different values of the auxiliary parameter $\varepsilon$, where the lower and upper limits within which the epsilon parameter must fall are obtained from the optimization of each separate scalar objective.

\section{Case study}

We consider the first example introduced by Guillén-Gosálbez and Grossmann (2009). This problem addresses the optimal retrofit of an existing SC established in Europe. There are 6 different technologies available to manufacture 6 main products in 2 plants, 2 warehouses and 4 final markets.

\section{Results and discussion}

The algorithm to construct the correlated multivariate distributions is implemented in MATLAB and provides the set of scenarios required by the GAMS optimization solver. Data is transferred to GAMS using the GDX facilities. The $\varepsilon$-constraint multi-objective 
optimization model was implemented in GAMS and solved to global optimality with CPLEX 12.5.1.0. The Life Cycle Inventory data for the raw materials, utilities used and transportation tasks have been taken from ecoinvent database (Hischier R., 2010). The optimization results have been post-processed with Matlab ${ }^{\circledR}$ using the GDXMRW suit of utilities to export/import data between GAMS and Matlab ${ }^{\circledR}$.

The results obtained shows how the correlation among environmental burdens affects the solution. To illustrate our results we present, as an example, one of the correlations studied. Among the three sources of environmental impact (raw materials, transport and utilities) considered in the supply chain design, raw materials exhibit the highest contribution to the GWP indicator. For the particular SC studied in this work, the most consumed raw material in the available technologies is propylene. Therefore, for illustration purposes, we investigate how the correlation among the burdens in the LCI of the raw material propylene, influences the stochastic design of the SC. For the calculation of the GWP in each scenario, the LCI considers 35 burdens, among which we correlated the two of them whose emissions provide the highest quantity of $\mathrm{CO}_{2}$ equivalent, while for the remaining burdens no correlation has been assumed. The two burdens with the highest contribution to the GWP for the raw material propylene are: carbon dioxide, fossil and methane, fossil. To further highlight the importance of using a stochastic model, we compared the solutions produced by the deterministic and stochastic approaches. Note that the deterministic model can be easily obtained from the stochastic one by defining only one single scenario which corresponds to the mean value of the uncertain parameter. Hence, we first solved the deterministic MILP maximizing the NPV and minimizing the WC of the GWP, thereby generating a set of SC designs and associated planning decisions. Then we incorporated the uncertain parameter and maximized the NPV and minimized the WC with the structural continuous and binary variables fixed to the values provided by the deterministic model. As seen in Figure 2, the stochastic solution dominates the deterministic design when we consider the two dimensional space given by the NPV and worst case.

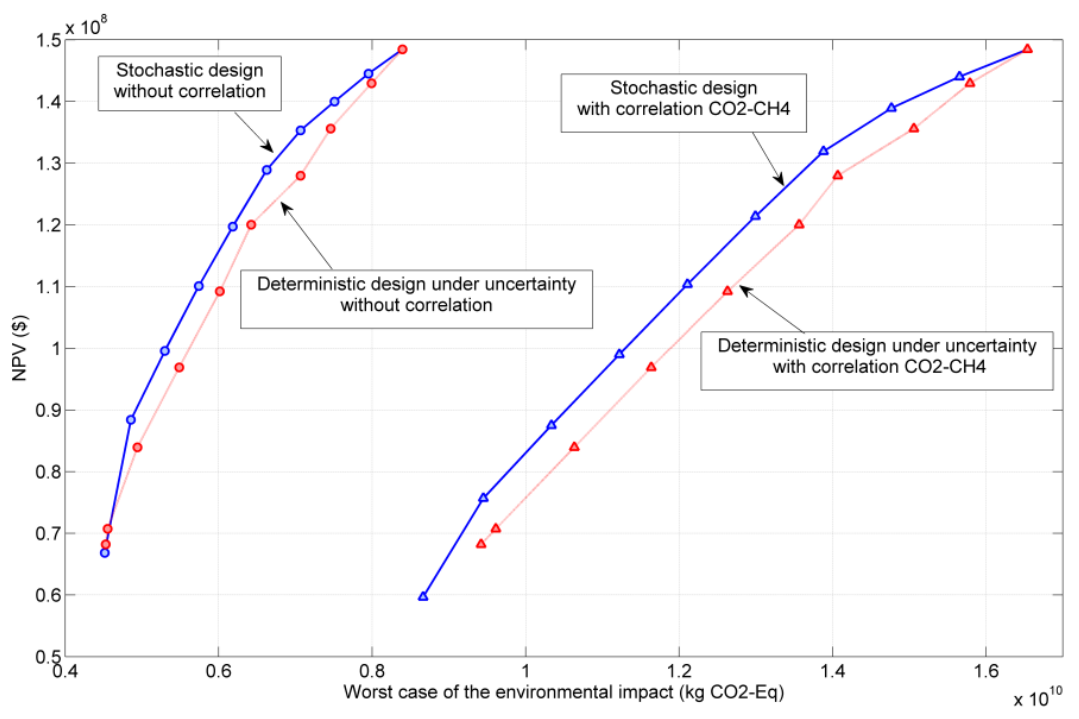

Figure 2. Pareto set of solutions for the deterministic and stochastic design with no burden correlation and correlating the two contaminants with more contribution to the GWP. 
Figure 2 also compares the solutions obtained when no correlation is applied for the design of the SC and when a correlation factor of 0.9 is applied to the selected burdens. The Pareto curves obtained considering correlation among LCI burdens are shifted to the right with respect to the Pareto curves without correlation. Hence, the latter curves represent too optimistic solutions, due to the fact that a non correlated uncertainty causes unrealistic scenarios where the contribution of a burden in the total environmental impact, is compensated by other burdens.

\section{Conclusions}

The results show how the incorporation of the correlation among the LCI entries that comprises the GWP, influences on the solution design of the supply chain. It has been proved that incorporating uncertainty in the LCI facilitates the decision-making process, providing a robust solution when burden emissions uncertainty reveals with different values from those nominal ones gathered in the environmental database.

\section{Acknowledgements}

The authors wish to acknowledge support from the Spanish Ministry of Science and Innovation (CTQ2012-37039-C02-02).

\section{References}

EHRGOTT, M. 2005. Multicriteria optimization, Berlin ; New York, Springer.

FRISCHKNECHT, R., JUNGBLUTH, N., ALTHAUS, H.-J., DOKA, G., DONES, R., HECK, T., HELLWEG, S., HISCHIER, R., NEMECEK, T., REBITZER, G. \& SPIELMANN, M. 2005. The ecoinvent Database: Overview and Methodological Framework (7 pp). The International Journal of Life Cycle Assessment, 10, 3-9.

GOEDKOOP, M., HOFSTETTER, P., MÜLLER-WENK, R. \& SPRIEMSMA, R. 1998. The ECO-indicator 98 explained. The International Journal of Life Cycle Assessment, 3, 352-360.

GROSSMANN, I. E. \& GUILLÉN-GOSÁLBEZ, G. 2010. Scope for the application of mathematical programming techniques in the synthesis and planning of sustainable processes. Computers \& Chemical Engineering, 34, 1365-1376.

GUILLÉN-GOSÁLBEZ, G. \& GROSSMANN, I. 2009. Optimal design and planning of sustainable chemical supply chains under uncertainty. AIChE Journal, 55, 99-121.

HISCHIER R., W. B., ALTHAUS H.-J., BAUER C., DOKA G., DONES R., FRISCHKNECHT R., HELLWEG S., HUMBERT S., JUNGBLUTH N., KÖLLNER T., LOERINCIK Y., MARGNI M., AND NEMECEK T 2010. Implementation of Life Cycle Impact Assessment Methods. Final report ecoinvent v2.2. Dübendorf, CH.: Swiss Centre for Life Cycle Inventories.

RUIZ-FEMENIA, R., GUILLÉN-GOSÁLBEZ, G., JIMÉNEZ, L. \& CABALLERO, J. A. 2013. Multi-objective optimization of environmentally conscious chemical supply chains under demand uncertainty. Chemical Engineering Science, 95, 1-11.

RUIZ-FEMENIA, R., SALCEDO-DÍAZ, R., GUILLÉN-GOSÁLBEZ, G., CABALLERO, J. A. \& JIMÉNEZ, L. 2012. Incorporating CO 2 emission trading in the optimal design and planning of chemical supply chain networks under uncertainty. Computer Aided Chemical Engineering, 30, 127-131. 